\title{
Method for Imaging the States of Water by Nuclear Magnetic Resonance in Low-water-containing Apple Bud and Stem Tissues
}

\author{
Merle M. Millard ${ }^{1}$, Dehua Liư ${ }^{2}$, Michael J. Line', and Miklos Faust ${ }^{2}$ \\ Beltsville Agricultural Research Center, Agricultural Research Service, Beltsville, MD 20705
}

\begin{abstract}
Additional index words. Malus domestica, proton spin density
Abstract. Magnetic resonance imaging estimates unreasonably high T2 times when creating T2 images in woody plants when tissues contain a limited amount of water. We developed a system to correct such images. Tissue distribution of proton density and states of water were determined by creating images of proton density and T2 relaxation times in summerdormant (paradormant) apple (Malus domestica Borkh.) buds. These images reveal that the proton density and water states obviously are not distributed uniformly in the bud and stem; but, the distribution of water depends greatly on the tissue type (bark, xylem, or meristem of the stem), and there are differences in the states of water even within the same tissue. At low proton density $\mathrm{T} 2$, calculated relaxation times were unreasonably high in tissues, with the exception of meristem of the shoot. In buds that were induced to grow and in which proton density was higher, T2 times appeared as expected. Variance of T2 times in tissues containing little water was 50 times higher than in those with a higher water content. Data with such high variance were excluded from the images; thus, the image was "corrected." Corrected images of T2 times fit the distribution of water indicated by the proton density images well.
\end{abstract}

Because of its non-invasive nature, nuclear magnetic resonance (NMR) has been used to investigate properties of water in biological systems (Aguyao et al., 1986; Belton and Ratcliffe, 1985), including plants (Gusta et al., 1979; Stout et al., 1978; Gassner, 1989 and Rajashekar et al., 1979). NMR determines the properties of water by measuring proton spin density and relaxation times by spectroscopy. These values are generally seen as averages in heterogeneous plant tissues; e.g., dogwood stem (Cornus florida Linn.) (Burke et al., 1975), ivy bark (Hedera helix Linn.) (Stout et al., 1978), maize roots (Zea mays Linn) (Basic and Ratkovic, 1987), tissue-cultured grape tissue (Abbas and Rajashekar, 1991), and wheat leaves (Triticum sp.) (Chen et al., 1978), among others. In determining relaxation times with NMR, the major problem is that calculated relaxation time involves a sum of contributions from all proton spins that may come from many environments of the heterogeneous plant tissue (Randall and Henkelman, 1986). The analysis of relaxation decay by the average sum of relaxation times may not reflect the water states of the tissue that play the crucial role in the plant response studied.

In more-recent studies, magnetic resonance imaging (MRI) was used to create images of specific tissues in apple buds (Faust et al., 1991), and in apple (Wang et al., 1988) pear (Pyrus communis Linn.) (Wang and Wang, 1989), and pelargonium hortorum $\times$ Bailey) roots (Brown et al., 1986) to be able to associate certain NMR-determinable characteristics visually with particular tissues. MRI is a somewhat unusual imaging technique in that, to a certain extent, the created image is a matter of choice. Thus, mobile 'proton density and spin-lattice (T1) or spin-spin (T2) relaxation times can be made the basis for the image (Morris, 1986).

Among the three choices mentioned above, T2 relaxation times have been used widely to describe the water states of tissues in both medical and plant studies (Gersonde et al., 1984; Morris, 1986; Rajashekar and Burke, 1986). In a few studies, T1 times also were

Received for publication 15 Sept. 1992. Accepted for publication 17 Jan. 1993. The cost of publishing this paper was defrayed in part by the payment of page charges. Under postal regulations, this paper therefore must hereby be marked advertisement solely to indicate this fact.

'Environmental Chemistry Laboratory.

${ }^{2}$ Fruit Laboratory. determined (Wang and Wang, 1991). However, in most studies, T1 or T2 relied on a single characteristic describing the state of water in the tissue. In our earlier study (Faust et al., 1991) involving dormancy of apple buds, the only major conclusion we could draw from spin-spin images was that the absence of an image evidenced strongly bound water and that a bright image, by comparison, indicated freer water.

Because calculation of relaxation times from sampling all the spins from many complex compartments of heterogeneous plant tissues may cause serious analysis errors, we believe that the best alternative is to derive relaxation images from spin echoes. This method allows direct analysis of comparison of the spatial distribution of relaxation times among different types of tissues, as Rumeur et al. (1987) suggested.

In studying the dormancy of the buds, we established that water is converted from bound to freer states in certain tissues of the bud when dormancy is broken (Faust et al., 1991). Water also moves into the bud when growth is resumed-a process that follows dormancy breaking by a period that could be days or weeks. The bud is a complex tissue and each component of the bud is very small. In establishing states of water and movement of water in specific tissues, visualization is essential by creating T2 and proton-density images. Best for visualization is production of the image in color; then, certain values are represented by a given color. Such color images indicate immediately the state the bud is in, what part of the bud water is bound or free, and what part of the bud water is penetrating if the bud is close to resumption of growth.

The possibility in creating images in woody tissues whose contrast or intensity is directly related to proton spin densitiesi.e., T1 and T2 effects-has been presented (Liu et al., 1993). During the course of this study, spin-spin, T2, proton spin density, and a variance image were generated from spin echo images using the program T2HVLINE. The T2, spin density, and variance images were constructed from a nonlinear least-square fit using the gradient descent method (Bevington, 1969) on a pel- (picture element) by-pel basis. T2 and spin density images are medianfiltered. The variance image was constructed pel by pel. Variance for each pel was estimated from image intensity decays by a single exponential. 


\section{Materials and Methods}

Although imaging plant tissues with MRI is done routinely, investigators experience problems in determining $\mathrm{T} 2$ times or obtaining an image in certain conditions accurately. Bowtell et al. (1990), using an 11.7T superconducting magnet, described dark areas in geranium (Pelargonium graveolens L'Her.) petioles that did not correspond to any real structure of the stem. The authors explained this deficiency by assuming a loss of signal caused by diffusion of water molecules in the very large magnetic field gradients that can occur close to the interphases between regions of differing magnetic susceptibility.

Morris et al. (1990), using a Bruker AM400 spectrometer for imaging castor bean (Ricinus communis zanzibariensis Linn.) seeds, noticed that the fit of the relaxation data to a single exponential was less than perfect. They shortened the echo time to improve the fit, but experienced the loss of signal from T2 decay, a major problem in the very short T2 systems. Kano et al. (1990) calculated T1 spin-lattice relaxation times and reported that the intensity of the signal was reduced by imaging pulse sequence with short repetition times.

We experienced poor fit of the $\mathrm{T} 2$ relaxation data to a single exponential when imaging dormant apple stems and buds, but fit was nearly perfect when buds were activated. Our echo delays were multiples of $11.2 \mathrm{~ms}$. Some investigators contend that this is too long (inappropriate) for measuring short $\mathrm{T} 2$ values. They contend that by using 11.2-ms echo time, the point acquired from the first echo point is the only significant signal and the system gives heavy weight to the long echo time data points. This contention is theoretically correct. There are laboratories that calculate $\mathrm{T} 2 \mathrm{val}-$ ues of 1-10 ms in leaves by using short echo times. However, we used 11.2-ms echo time to limit the gradient strength, to reduce diffusion effects, and to limit eddy currents. The 11.2-ms echo time gave us good anatomical separation of values. We also used 4-ms pulse sequences for sharp slice selections. Working with small structures, such as an axis of a bud, sharp slice selection is essential.

Shortening echo time for increasing accuracy of measuring very short $\mathrm{T} 2$ times $(<6 \mathrm{~ms})$ was less important, in our case, than associate $\mathrm{T} 2$ times in the higher range (i.e., 15 to $35 \mathrm{~ms}$ ) with the anatomical structure of the stems and buds. We wanted to distinguish between bound $(<6-10 \mathrm{~ms})$ and free water $(20-30 \mathrm{~ms})$ and the exact value of bound water did not matter as long as we knew it was bound. Therefore, we used a relatively long echo $(11.2 \mathrm{~ms})$ and developed a system that disregarded values of $\mathrm{T} 2$ times with a high variance.

For MRI imaging, we placed shoots into 10-mm NMR tubes (Faust et al., 1991). Image slices were taken perpendicular to the axis of the shoot through the midpoint of buds (Fig. 1). For imaging, a Bruker MSL $400 \mathrm{MHz}$ (9.4-T) instrument (Bruker Instrument Co., Manning Park, Bellerica, Mass.) was used. Single- slice multi-echo images were collected using a standard multi-echo imaging pulse sequence. Spin echo images with dimensions of $256 \times 256$ pels were obtained whose spatial resolution was $29.3 \times$ $29.3 \times 500 \mu \mathrm{m}$. We used a $4-\mathrm{ms} 90^{\circ}, 50-\mathrm{kHz}$ sweep-width pulse sequence. Acquisition with maximum desired gradient was 125 $\mathrm{mT} / \mathrm{m}$. Repetition time was $2 \mathrm{~s}$ and echo delays were multiples of $11.2 \mathrm{~ms}$. Images were transferred from the Aspect 3000 computer of the imaging instrument to Sun SPARC II work stations via Ethernet communications. Images were reconstructed using MRI/ IMAGE analysis software (New Methods Research Inc., East Syracuse, N.Y.). Proton spin density and T2 images were generated using a computer program written by M.J. Line called T2HVLINE.

Images were produced in color. A different color is assigned to

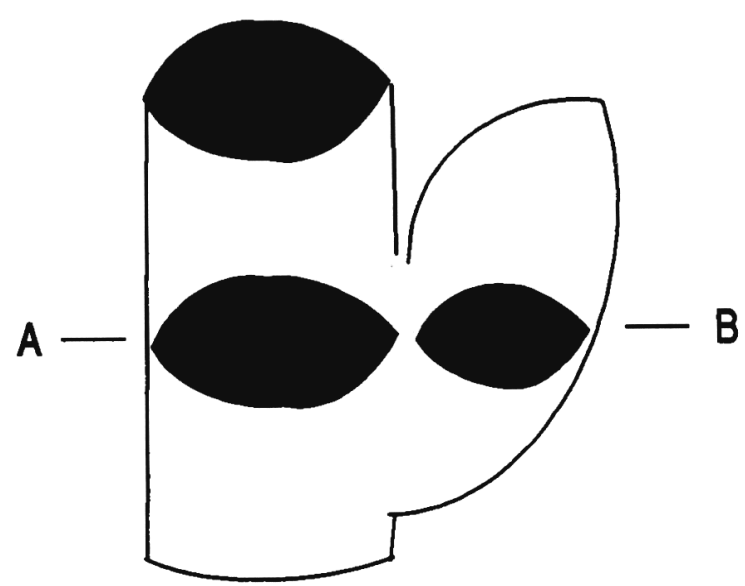

Fig. 1. Schematic illustration of MRI image through apple stem and bud. Imaging was done in the plant A-B.

a range of proton spin density and $\mathrm{T} 2$ times; thus, an image is created associating a given range of values with a specific area within the tissue. T2 values are colored in unequal steps up to 100 ms: dark red, <10; red, 11-15; yellow, 16-20; blue, 21-25; green, 26-30; maroon, 31-35; pink, 36-50; and purple, 51-100 ms. Proton spin density is expressed as an arbitrary equally spaced scale from 1 to 8 from pink (low) to white (high).
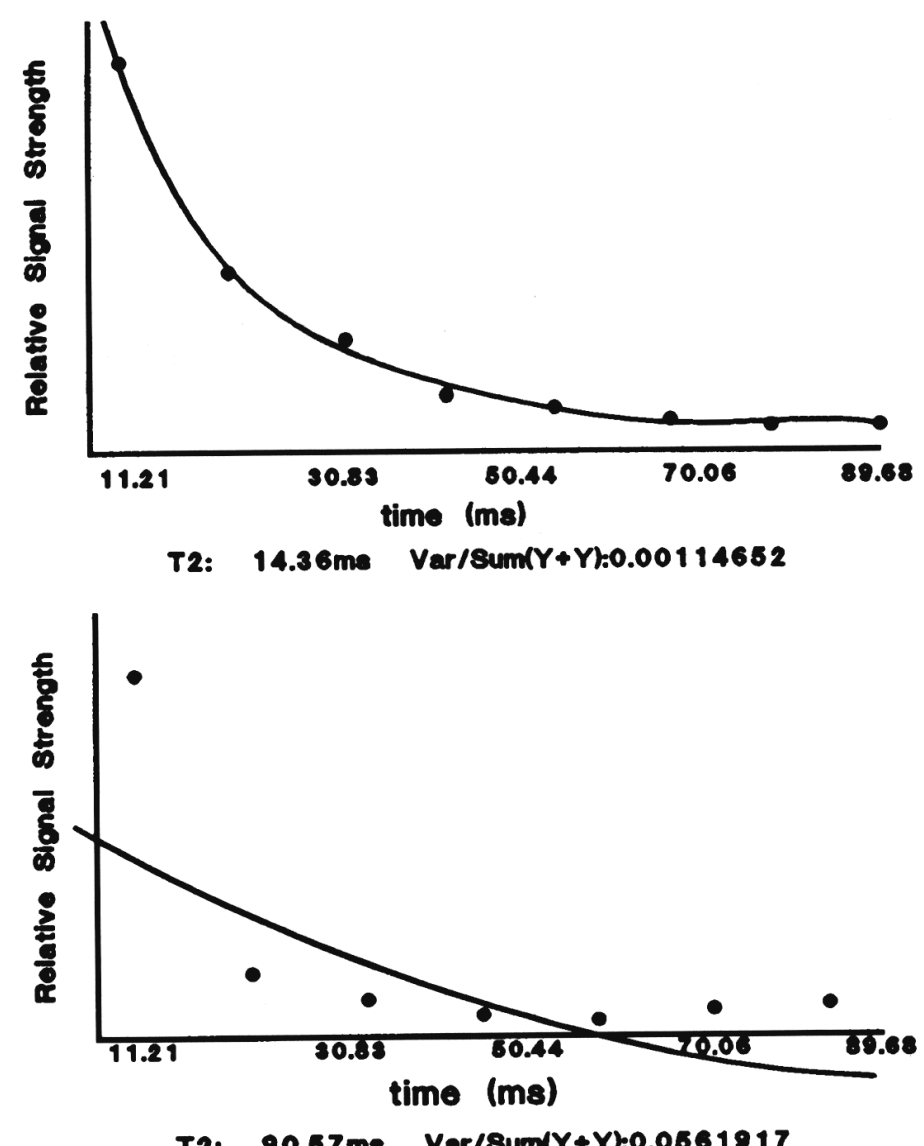

T2: $90.67 \mathrm{me}$ Var/8um(Y+Y):0.0661917

Fig. 2. Examples of nonlinear square fitting of echo points to determine T2 time Top: Fit with a relatively low variance obtained in tissues where proton density signals are high. Bottom: Fit with a relatively high variance obtained in tissues where proton density signals are low. Note the rapid decrease between echos 1 and 2 , given in milliseconds, and the relative flat nature of the curve between echoes 2 and 8 . 

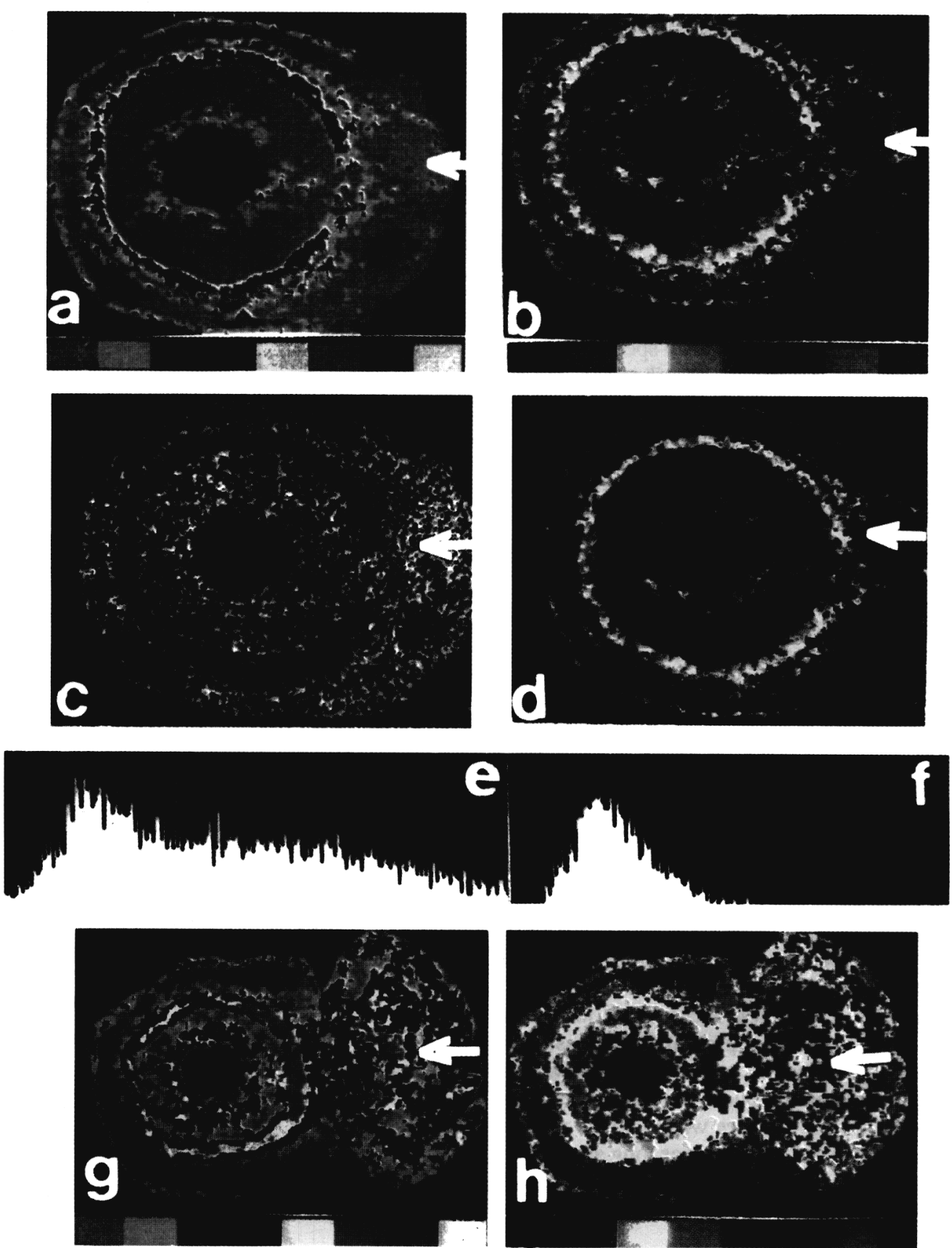

Fig. 3. Various images of stem and bud sections. Location of bud is indicated by arrows, the ring structure in the stem is the meristematic tissue. (a) Proton density image of a dormant bud. Color code is an arbitrary scale from low (pink) to high (white) in eight increments. (b) Uncorrected T2 image of the bud in (a). Color code is as follows: dark red, <10; red, 11-15; yellow, 16-20; blue, 21-25; green, 26-30; maroon, 31-35; pink, 36-50; and purple, 51-100 ms. (c) Error signal distribution for T2 image. Pixels with high variance values are indicated on the image. Note that the high variance is absent in the meristematic tissue (black ring corresponding to green ring in (a) and yellow ring in (b). (d) Corrected T2 image. All T2 values with high variance were eliminated from the image presented in (b). Color coding is the same as for (b). (f) Histogram of signals of the image presented in (d). (g) Proton density image of a bud $48 \mathrm{~h}$ after it was induced to grow. Note the influx of water indicated by higher proton density signals. Color coding is the same as (a). (h) T2 image of a bud $48 \mathrm{~h}$ after it was induced to grow. Color coding is the same as for (b).

\section{Results and Discussion}

In some images where proton density signals are very low, the T2 times do not represent a valid value. Nonlinear least-square fitting of the eight echo points indicates clearly the difference in fit of a curve when proton density signals (water content) is sufficiently high (Fig. 2, top) and when proton density signals are weak (water content is low) (Fig. 2, bottom). The fit is good in the first case and poor in the second. The variance is nearly 50 times greater for the poor tit, resulting in unrealistically high $\mathrm{T} 2$ values.

In our images the signal echo time (te) was $\approx 11.21 \mathrm{~ms}$, which is about the minimum for producing clear T2 images in woody tissues. This means that any T2 that is shorter than $\approx 6 \mathrm{~ms}$ will result in a very large error. Depending also on the proton density, the T2 value will be greatly overestimated. This situation differ from that of spectroscopic T2 measurements where te can be as short as 100 $\mu \mathrm{s}$, and components with much shorter T2 times can be measured. Therefore, images were corrected by developing a variance image (Fig. 3c) and eliminating T2 values with large variance from the corrected T2 image (Fig. 3d). The variance was considered too large if it exceeded $>1 \%$ of the intensity. The corrected T 2 image (Fig. 3d) correlates well with the proton density image (Fig. 3a). 
Even though many T2 values were eliminated from the image, those that remained correspond to the meristematic (cambial) ring where the water concentration, indicated by the proton density image, was higher. The overall bud water content ranged around $50 \%$, but, because of the difficulty in separating the tissues and their small volume, the actual water content for each tissue type could not be determined. Signal distribution further amplifies the need for correction of $\mathrm{T} 2$ images. The uncorrected $\mathrm{T} 2$ signal distribution (Fig. 3e) is wide and may appear as a composite of several populations of signals. When signals with high variance are eliminated from the $\mathrm{T} 2$ image, the $\mathrm{T} 2$ signal distribution is greatly sharpened (Fig. 3f). In buds that were induced to grow and that contained sufficiently high concentration of water, indicated by stronger signals in the proton density images (Fig. 3g), T2 times seem to be in a reasonable range and the $\mathrm{T} 2$ images do not need correction (Fig. 3h).

To know the correctness of T2 values in woody plants where water content is low, both proton spin density and T2 time images should be constructed. If proton spin density is low and T2 time is high in a given tissue, the T2 time is in error and should be disregarded. In our example, when an area is pink (lowest water content) on the proton density image and purple (highest T2 value) on the T2 image, the T2 value in reality should be very low and in all likelihood it will not create an image. For practicality, it is simpler to disregard certain areas of the image when colors (values) of low proton density and high T2 coincide than to construct variance images and a corrected T2 image. However, when high accuracy is required, this should be done.

\section{Literature Cited}

Abbas, M. and C.B. Rajashekar. 1991. Characterization of heat injury in grapes using nuclear magnetic resonance. Plant Physiol. 96:957-961.

Aguyao, J.B., S.J. Blackband, J. Schoeninger, M.A. Mallingy, and M. Hinterman. 1986. Nuclear magnetic resonance imaging of a single cell. Nature (London) 322:190-191.

Basic, G. and S. Ratkovic. 1987. NMR studies of a radial exchange and distribution of water in maize roots: Relevance of modeling of exchange kinetics. J. Expt. Bot. 38:1284-1297.

Belton, P.S. and R.C. Ratcliffe. 1985. NMR and compartmentalizes in biological tissues. Prog. in NMR Spectr. 17:241-279.

Bevington, P.R. 1969. Data reduction and error analysis for the physical sciences. McGraw-Hill, New York.

Bowtell, R.W., G.D. Brown, P.M. Glover, M. McJury, and P. Mansfield. 1990. Resolution of cellular structures by NMR microscopy at $11.7 \mathrm{~T}$. Proc. Trans. R. Soc. London (Ser. A). 333:457-467.

Brown, J.M., G.A. Johnson, and P.J. Kramer. 1986. In vivo magnetic resonance imaging of changing water content in Pelargonium hortorum roots. Plant Physiol. 82:1158-1160.

Burke, M.J., R.G. Brandt, and C.J. Weiser. 1974. Nuclear magnetic resonance of water in cold-acclimating 'Red Osier' dogwood stem. Plant Physiol. 54:392-398.

Chen, P.M., L.V. Gusta, and D.G. Stout. 1978. Changes in membrane permeability of winter wheat cells following freeze-thaw injury as determined by nuclear magnetic resonance. Plant Physiol. 61:878-882.

Faust, M., D. Liu, M.M. Millard, and G.W. Stutte. 1991. Bond versus free water in dormant apple buds-A theory for endodormancy. HortScience 26:887-890.

Gassner, G. 1989. Magnetic resonance imaging in agriculture, p. 405428. In: P.E. Pfeffer and W.V. Gerasimowicz (eds.). Nuclear magnetic resonance in agriculture. CRC Press, Boca Raton, Fla.

Gersonde, K., L. Felsberg, T. Tasfdorff, D. Ralzel, and B. Sterokel. 1984. Analysis of multiple $\mathrm{T} 2$ proton relaxation processes in human head and imaging on the basis of selective and assigned T2 values. Magn. Reson. in Med. 1:463-477.

Gusta, L.V., D.B. Fowler, P. Chen, and D.G. Stout. 1979. NMR study of water in cold-acclimating cereals. Plant Physiol. 63:627-634.

Kano, H., N. Ishida, T. Kabayashi, and M. Koizumi. 1990. 1H-NMR imaging analysis of changes in free water distribution in barley and soybean seeds during maturation. Jpn. J. Crop Sci. 59:503-509.

Kuhn, W. 1990. NMR microscopy fundamentals, limits and possible applications. Angewandte Chemie Int. 29: 1-112.

Liu, D., M. Faust, M.M. Millard, M.J. Line, and G.W. Stutte. 1993. States of water in dormant and activated summer apple buds determined by proton magnetic resonance imaging. J. Amer. Soc. Hort. Sci. 118:632637.

Morris, P.G. 1986. Nuclear magnetic resonance imaging in medicine and biology. Clarendon Press, Oxford, U.K. p. 270-279.

Morris, P.G., H.E. Darceuil, A. Jasinski, A.K. Jha, D.J.O. McIntire, and D. H. Northcote. 1990. NMR microscopy of the germinating castor bean. Phil. Trans. R. Soc. London (Ser. A). 333:487-493.

Rajashekar, C.B., L.V. Gusta, and M.J. Burke. 1979. Membrane structural transitions. Probable relation to frost damage in hardy herbaceous species, p. 255-274. In: J.M. Lyons, D. Graham, and J.K. Raison (eds.). Low temperature stress in crop plants. Academic, New York.

Randall, M.K. and R.M. Henkelman. 1986. Analysis of biological NMR relaxation data with continuous distribution of relaxation times. J. Magn. Reson. 69:218-235.

Rumeur, E.L., J.D. Certaines, P. Toulouse, and P. Rochcongar. 1987. Water phase in rat striated muscles as determined by T2 proton NMR relaxation times. Magn. Res. Imaging 5:267-272.

Stout, D.G., P.L. Steponkus, and R.M. Cotts. 1978. Nuclear magnetic resonance relaxation times and plasmolemma water exchange in ivy bark. Plant Physiol. 62:636-641.

Wang, C.Y. and P.C. Wang. 1989. Nondestructive detection of core breakdown in Bartlett pears with nuclear magnetic imaging. HortScience 24:106-109.

Wang, C.Y. and P.C. Wang. 1991. Nuclear magnetic resonance imaging of chilled and nonchilled zucchini squash. HortScience 26:697. (Abstr.).

Wang, S.Y., P.C. Wang, and M. Faust. 1988. Non-destructive detection of watercore in apple with nuclear magnetic resonance imaging. Scientia Hort. 35:227-234. 